\title{
Observatorio
}

\section{Servicios de referencia para una sociedad digital}

\author{
Por José-Antonio Merlo-Vega
}

\begin{abstract}
Resumen: Los servicios de referencia han evolucionado al mismo ritmo que las tecnologías. Se presentan diferentes aspectos de la sociedad de la información relacionados con los servicios de consulta que prestan bibliotecas y centros de información. Se describen las condiciones de ubicuidad, inmediatez y participación que definen a la sociedad digital. La referencia digital forma parte de la sociedad de la información. Se reflexiona sobre la evolución de los servicios de consulta que ofrecen los centros de información. Se concluye planteando las características de la referencia digital: servicios telemáticos, colecciones híbridas y profesionales competentes.

Palabras clave: Referencia digital, Sociedad de la información, Cultura digital, Servicios de información.
\end{abstract}

\section{Title: Reference services for a digital society}

Abstract: Reference services have developed at the same pace as technology. Different aspects of the information society related to reference services provided by libraries and information centres are presented, including a description of the ubiquity, immediacy and participation that define the digital society. Digital reference is part of the information society and reflects the evolution of reference services offered by information centres. The characteristics of digital reference are described: telematic services, hybrid collections and skilled professionals.

Keywords: Digital reference, Information society, Digital culture, Information services.

Merlo-Vega, José-Antonio. "Servicios de referencia para una sociedad digital”. El profesional de la información, 2009, noviembre-diciembre, v. 18, n. 6, pp. 581-586.

DOI: 10.3145/epi.2009.nov.01

\section{La sociedad digital}

\section{VIVIMOS EN UNA SOCIEDAD DIGITAL. Nos} relacionamos a través de redes que conectan miles de máquinas, que facilitan millones de datos.

Convivimos de forma natural con dispositivos electrónicos que empleamos indistintamente con fines personales o profesionales. Utilizamos sistemas de comunicación basados en protocolos de correo, medios web o redes telefónicas. Recurrimos a las redes para localizar datos y documentos. Formamos parte de la cultura digital. Pasado, presente y futuro se mezclan en una sociedad donde los procedimientos tradicionales se simultanean con las técnicas más avanzadas.

Así ocurre en las unidades de información. Los servicios en las bibliotecas y los centros de información han evolucionando al ritmo de los avances tecnológicos, de la progresiva "digitalización" de la sociedad, de la aparición de nuevas aplicaciones que se utilizan para la prestación de viejos servicios o para la creación de otros innovadores.

La evolución de los servicios digitales en los centros de información debe entenderse como una faceta más de la conversión de la sociedad industrial en sociedad de la información, en la sociedad digital. Administración, economía, cultura, educación, información... todos los ámbitos sociales actúan ya a partir de una base tecnológica, sustentándose en equipos, programas y redes. La aplicación de las tecnologías a cualquier faceta de la actividad humana hace que se pueda hablar de la presente sociedad digital que, entre otros aspectos, puede identificarse mediante tres rasgos definitorios: ubicuidad, inmediatez y participación ${ }^{1}$.

Gracias a la expansión de las redes wi-fi y telefónicas, la disponibilidad de cobertura es amplia, lo que hace posible que un dispositivo pueda obtener conexión desde cualquier lugar. A esto se une una nueva genera- 
ción de aparatos destinados a usos múltiples (telefonía, internet, multimedia), mediante los cuales es posible conectarse a una red desde cualquier punto.

Además la sociedad digital permite que un dato o un documento que se necesite puedan ser obtenidos de forma inmediata. La conexión permanente y la oferta de contenidos y servicios posibilitan que las necesidades de información puedan ser satisfechas en el instante.

Por último, la sociedad digital es altamente participativa. Estamos presentes en redes sociales, nuestros datos se comparten con entidades, valoramos informaciones, aportamos opiniones, sobre todo, creamos contenidos de forma conjunta. En la sociedad digital las personas actúan como el eje central que da valor y se superpone al trinomio equipos, programas y redes. Las tecnologías de la información (máquinas y aplicaciones) y las tecnologías de las comunicaciones (redes) se ven hoy completadas por las tecnologías de la participación (personas), aquéllas que se agrupan bajo la denominada web social o web 2.0.

\section{"La evolución de los servicios digitales debe entenderse como una faceta más de la conversión de la sociedad industrial en la sociedad de la información"}

Las tecnologías permiten que una persona que necesita una información, la obtenga en el momento y en el lugar en que se encuentre. Pero, para que dicha información esté accesible, alguien tiene que facilitarla y alguien tiene que ayudar a encontrarla si el usuario no dispone de conocimientos o recursos para hacerlo de forma autónoma. Los centros de información siempre han cumplido esa función y hoy más que nunca sigue siendo necesaria la figura del experto al que acudir en busca de un dato o un documento. La sociedad de la información necesita profesionales de la información y éstos solamente lo serán si, además de otros conocimientos y destrezas, tienen competencias tecnológicas.

Los profesionales de las bibliotecas y los centros de información sólo podrán ser útiles a una sociedad digital si, además de los perfiles formativos profesionales tradicionales, disponen de la suficiente educación tecnológica. Los servicios de información y referencia resultarán eficaces en una sociedad digital únicamente si pueden ser empleados desde cualquier lugar y en cualquier momento. Por este motivo, es necesario en los sistemas de referencia el uso de herramientas que permitan la comunicación sincrónica con el usuario.
Las posibilidades tecnológicas para los servicios de información y referencia son amplias. De hecho, los sistemas de información en línea están empleando las diferentes opciones que en la actualidad aporta la tecnología: correo electrónico, formularios web, chat, mensajería instantánea, telefonía IP, videoconferencia, listas de correo o noticias, redes sociales, blogs, mensajería móvil o aplicaciones propietarias. A medida que se han ido produciendo avances tecnológicos, los centros de información los han ido integrando en la prestación de sus actividades, modificando sus procesos o, incluso, creando nuevos servicios.

\section{"Las tecnologías de la información (máquinas y aplicaciones) y las tecnologías de las comunicaciones (redes) se ven hoy completadas por las tecnologías de la participación (personas)"}

Los servicios de información y referencia son, precisamente, los que en mayor medida se han beneficiado de las posibilidades que ofrece la aplicación de tecnologías a los centros de gestión del conocimiento. Aumentan los recursos, se recorta el período de respuesta; crece el número de usuarios, se multiplica el alcance. La expansión de la banda ancha para la transmisión de datos permite ofrecer servicios telemáticos en tiempo real, conversando con el usuario independientemente de su ubicación, navegando con él en busca de la información necesitada, convirtiendo en cercanía la posible distancia entre quien realiza la consulta y quien le proporciona la respuesta. Además, la tendencia a implementar aplicaciones interoperables está permitiendo que los servicios de información empleen las tecnologías de forma creativa, adaptando a sus posibilidades y necesidades la oferta existente. Es común emplear widgets, reutilizar contenidos o por ejemplo, integrar APIs en webs. La personalización y adaptación de tecnologías o aplicaciones hacen que cualquier centro pueda ofrecer servicios de información y referencia, aunque disponga de escasos recursos para ello.

\section{La referencia digital}

La investigación en bibliotecología suele afirmar que la referencia, como campo de estudio, nace en 1876, cuando Library Journal publica el artículo de Samuel Swett Green "Personal relations between librarians and readers", en el que se defiende la función informativa de la biblioteca ${ }^{2}$. De igual forma, se toma 1984 como el año en el que se inicia el primer servicio 


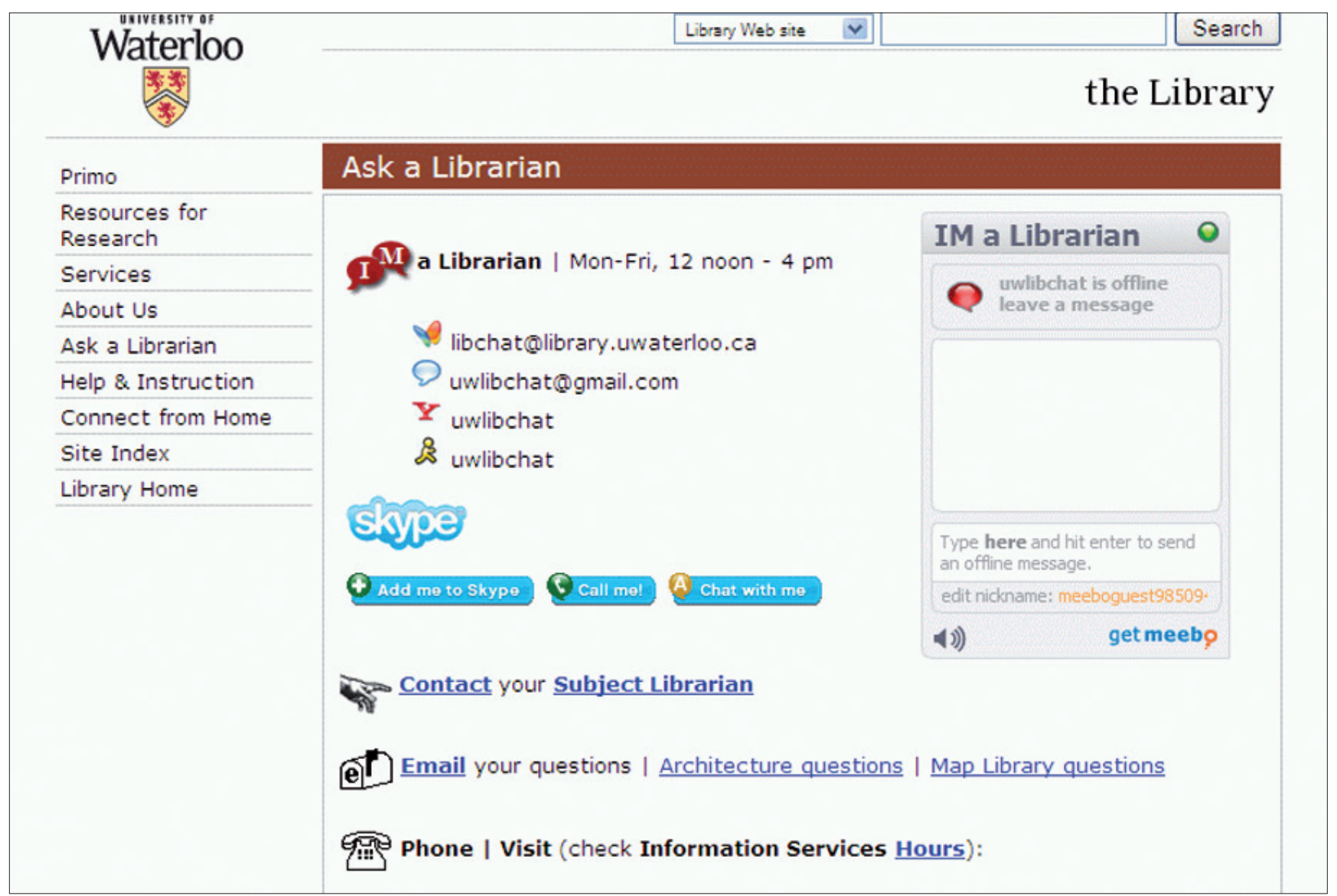

Figura 1. Los servicios de referencia digital ofrecen diferentes tecnologías para la consulta (University of Waterloo Library, Ontario, Canada) http://www.lib.uwaterloo.ca/asklib/

de referencia digital, el denominado Electronic Access to Reference Services (EARS), de la Maryland's Health Services Library ${ }^{3}$. Se trata de una realidad joven, ya que es en la década de los 90 cuando se ponen en práctica los servicios de consulta en internet. Las primeras iniciativas se basaron únicamente en la posibilidad de enviar preguntas a una biblioteca o un centro mediante correo electrónico, pero rápidamente la referencia digital va tomando la forma actual delimitando su actividad, estableciendo sus procedimientos y aplicando las tecnologías en función de los objetivos que se pretendan alcanzar.

\section{"La tendencia a implementar aplicaciones interoperables está permitiendo que los servicios de información empleen las tecnologías de forma creativa"}

En un sentido amplio, se debe entender la referencia digital como el servicio de consulta que proporcionan los centros de información en internet. En su sentido más estricto, se debe limitar este servicio a la posibi- lidad que ofrecen bibliotecas y centros de información para que se les planteen consultas de información concreta o asesoramiento documental, empleando medios telemáticos para la comunicación y para la transferencia de información.

La referencia digital es, por tanto, un servicio ofrecido por centros de información, donde la comunicación con los usuarios se realiza mediante redes telemáticas y en el que los datos o documentos se sirven electrónicamente. La referencia digital es un servicio profesional, telemático y electrónico. Las particularidades asignadas en párrafos anteriores a la sociedad digital se pueden aplicar también a la referencia digital, ya que se trata de un servicio ubicuo, en el que no importa la distancia entre quien pregunta y quien responde; incluso, puede tratarse de una actividad realizada a modo de teletrabajo, desde los propios domicilios de los profesionales de la información. Los servicios siguen siendo necesarios, los espacios presenciales ya no.

Del mismo modo, la referencia digital es -o puede ser- un servicio inmediato, puesto que la relación usuario-referencista puede establecerse en tiempo real, tanto para realizar la entrevista de referencia o delimitación de la consulta, como para localizar la información demandada, valorarla y ofrecerla al usuario. 
Si otra de las propiedades de la sociedad digital es la participación, lo mismo puede decirse de la referencia digital, que suele ser cooperativa y en la que los usuarios pueden aportar respuestas o completar las ofrecidas por los servicios de información.

\section{"La referencia ya no podría realizarse fuera de los entornos digitales"}

La referencia ya no podría realizarse fuera de los entornos digitales. La adaptación ha sido rápida y eficaz. Los centros de información basan en las tecnologías sus sistemas de referencia inmediata o diferida, ya sea combinando esta tarea con servicios presenciales o bien creando un nuevo servicio de información telemática. En todo caso se trata de servicios y, por lo tanto, es necesario establecer políticas, normalizar procedimientos y disponer de personal especializado.

En primer lugar, una biblioteca o un centro de información que desee ofrecer referencia digital debe aprobar una política de prestación de este servicio, en la que se delimiten los objetivos, los destinatarios, los referencistas, las tecnologías, los recursos, la administración y la evaluación del mismo.

Para establecer una política y su carta de servicios, son útiles las recomendaciones de autores como Berube $^{4}$ o Nguyen ${ }^{5}$, así como las directrices aprobadas por $I F L A^{6}$ y RUSA ${ }^{7}$. Merlo, en su manual Información y referencia en entornos digitales ${ }^{8}$, sistematiza los elementos que hay que determinar para la prestación de la referencia digital y proporciona una relación de cincuenta recomendaciones que hay que tener en cuenta a la hora de planificar y mantener este servicio. Se trata de una lista de directrices que pueden servir de pautas para el diseño y organización de servicios de referencia digital o bien como lista de comprobación para cerciorarse de que se han tenido en cuenta todos los elementos que se conjugan en la prestación telemática de servicios de consulta. Las políticas son necesarias ya que, coincidiendo con Kern ${ }^{9}$, hacen que los usuarios conozcan qué pueden esperar del servicio. Las políticas de referencia delimitan quién puede usar el servicio de referencia digital, qué tipo de preguntas se pueden realizar, cómo se realizará el proceso de consulta/respuesta o cuándo se obtendrá la información solicitada.

Un aspecto esencial es el perfil del personal que atiende las consultas de los usuarios. Las competencias del referencista digital han sido uno de los temas más profusamente tratados en la literatura profesional. Kovacs ${ }^{10}$ es quien mejor expone esta cuestión, esta- bleciendo una extensa relación de destrezas que debe poseer un profesional de la información que desempeñe su actividad en servicios de referencia. Esta autora organiza las competencias en tres grupos: técnicas, comunicativas y de referencia. Las competencias técnicas se refieren a los conocimientos relativos a tecnologías, uso de ordenadores y periféricos, manejo de aplicaciones informáticas y transferencia de información por medios electrónicos. Las comunicativas inciden en las habilidades para relacionarse con los usuarios, para entender sus consultas, para delimitar sus necesidades de información y para seguir en todo momento pautas de comportamiento social. Por último, las de referencia reúnen las capacidades y destrezas del personal para localizar recursos de información, para trabajar con los mismos y para proporcionar al usuario el dato o documento que necesite.

\section{"Un aspecto esencial de la referencia digital es el perfil del personal que atiende las consultas de los usuarios"}

\section{La referencia digital no es virtual}

La referencia digital es real, por eso en las líneas anteriores se ha evitado el calificativo "virtual". Todavía en castellano lo virtual se corresponde con el efecto de asemejar una realidad que no existe. Cierto es que, desde que existe internet, lo virtual es sinónimo de lo telemático, de aquella actividad que se realiza en línea. La aparición de las primeras colecciones de recursos y de servicios en red trajo consigo el concepto de bibliotecas virtuales, a pesar de tratarse de centros reales, que seleccionan colecciones, que las organizan y que las ofrecen a sus usuarios, como cualquier biblioteca.

Los servicios de información y referencia digital también son reales: hay una comunicación entre personas, que se relacionan independientemente de los espacios físicos en los que se encuentren, que intercambian información y donde se realiza una transacción de datos o documentos. En servicios de información, se emplean indistintamente los términos "digital" y "virtual", algo que no es erróneo, aunque sí es necesario entender que el concepto de referencia digital es más preciso (documentos y conexiones electrónicos) que el de referencia virtual, que sólo alude a que se trata de un servicio de consulta que se presta en línea.

La perspectiva de partida de este Observatorio ha sido insertar la referencia digital en una sociedad en la que se convive con las tecnologías, que las ha asimilado de manera espontánea, de tal forma que muchas de 


\section{bibliotek $^{\text {suar }}$}

\section{Have a question?}

Contact your local public library when you need to know if a specific book is on the shelf, or when you have questions about reservations or hours of business.

$\underline{\text { Read more }}$

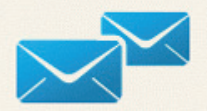

\section{E-POST}

Send queries around the clock you'll receive an answer by e-mail in the course of the next business day.

$\underline{\text { Read more }}$

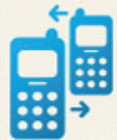

SMS

Send svar and your question to 1980. You'll receive an answer in the course of the next business day

$\underline{\text { Read more }}$

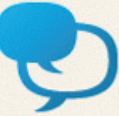

\section{CHAT}

Open workdays $10-16.00$ (15.30 fridays). Use the chat for simple information needs. The chat is closed.

$\underline{\text { Read more }}$

Figura 2. Los servicios públicos de referencia digital están abiertos a cualquier usuario (Biblioteksvar, Noruega) http://biblioteksvar.no/en/

las actividades sociales se realizan mediante equipos y redes. Los centros de información deben seguir esta estela y de hecho lo están haciendo. Las bibliotecas y las unidades de información saben que el usuario puede necesitar sus servicios en cualquier momento y desde cualquier lugar, por lo que le facilitan medios para que sus demandas de información queden recogidas y puedan ser resueltas. Es posible concluir esta reflexión sintetizando en tres grandes aspectos los elementos que definen la referencia digital, que la identifican tal y como se está prestando en la actualidad:

\section{"Los servicios de referencia digital son la materialización de cómo las bibliotecas y las unidades de información se están integrando en la sociedad de la información, en el mundo digital”"}

- Servicios telemáticos. Se sustenta en redes de telecomunicaciones. Hasta la propagación de internet no existieron servicios de este tipo. La informática y las telecomunicaciones son mucho más antiguas, pero el concepto de referencia digital va estrechamente unido al de internet. Los grandes servicios públicos de referencia digital nacieron a finales de los años 90 y muchos de ellos incluso en el siglo XXI y lo mismo ha ocurrido en las universidades y las bibliotecas especializadas, que comienzan a proporcionar esta prestación a finales del siglo pasado y principios del presente. Los servicios de referencia digital han hecho de la telemática su principal base para poder emplear tecnologías de mensajería instantánea, chat, telefonía IP, redes sociales, correo electrónico o formularios web.
- Colecciones híbridas. Emplea documentación en formato electrónico en un porcentaje muy elevado, lo cual no implica que sea el único tipo de colección que utiliza. Como en la referencia tradicional, los fondos de referencia son mixtos, ya que será tan importante una fuente impresa, como un audiovisual o un dato extraído de un recurso en línea. No obstante, los recursos digitales son los más empleados, dada la rapidez que muchas veces se necesita en un servicio de referencia digital. Por ello, hay que dominar el uso de buscadores, disponer de metabuscadores en las bibliotecas y servicios especializados y mantener selecciones organizadas de recursos de información.

- Profesionales competentes. Necesita ser administrada por especialistas, debe ser desempeñada por referencistas competentes, formados en biblioteconomía, expertos en tecnologías (aquí no sirve el "nivel de usuario"), preparados para dirigir relaciones en línea y conocedores de los recursos de información: dónde está la información y cómo se accede a ella. No es redundante insistir en que un referencista digital basará su trabajo en el uso de tecnologías, por lo que los conocimientos informáticos son indispensables. Del mismo modo, prestará un servicio público, por lo que debe saber cómo comunicarse, teniendo un ordenador como intermediario. Además, facilita un servicio de información, por lo que, para que exista calidad en la resolución de las demandas, debe disponer de conocimientos, destrezas y experiencia en el manejo de fuentes de referencia.

Ubicuidad, inmediatez, participación. Servicios telemáticos, colecciones híbridas, profesionales competentes. Los servicios de referencia digital son la materialización de cómo las bibliotecas y las unidades de información se están integrando en la sociedad de la información, en el mundo digital, en el que las re- 
laciones personales y las profesionales no necesitan cercanía para ser reales. Los servicios de información no deben limitarse a un espacio y un horario concretos. Los usuarios tienen necesidades de información y emplean cotidianamente dispositivos mediante los que hacer llegar esas consultas a especialistas, a los centros de información.

\section{"Los servicios de información no deben limitarse a un espacio y un horario concretos"}

Resolver consultas y asesorar en documentación es algo que saben hacer bien los profesionales. La referencia digital aporta que la relación con el usuario sea constante: si hay una pregunta, hay también servicios de referencia digital para responderla.

\section{Notas}

1. Estos tres aspectos son analizados en Merlo-Vega, José-Antonio. Información y referencia en entornos digitales: desarrollo de servicios bibliotecarios de consulta. Murcia: EDITUM, 2009, capítulo 11.
2. Tyckoson, David A. "What is the best model of reference service?" $\mathrm{Li}$ brary trends, 2001, v. 50, n. 2, p. 183-196.

3. Kresh, Diane. "Virtually yours: thoughts on where we have been and where we are going with virtual reference services in libraries". The Reference Librarian, 2002-2003, v. 38, n. 79/80, p. 19-34.

4. Berube, Linda. Digital reference overview. Bath: UKLON, Networked Services Policy Task Group, 2003.

5. Nguyen, Claire. Mettre en place et développer un service de référence virtuelle dans une perspective d'integration à un réseau collaboratif: Analysis comparées et propositions à partir de l'exemple de l'Université de Montréal: Mémoire d'étude. Villeurbanne: ENSSIB, 2006.

6. IFLA. Recomendaciones para el servicio de referencia digital. Madrid: ANABAD, 2004

http://archive.ifla.org/VII/s36/pubs/drgO3-s.htm

7. RUSA. Guidelines for implementing and maintaining virtual reference services. Chicago: ALA, RUSA, 2004.

http://www.ala.org/ala/mgrps/divs/rusa/resources/guidelines/virtrefguidelines. cfm

8. Merlo-Vega, José-Antonio (2009), op. cit., capítulo 8.

9. Kern, M. Kathleen. Virtual reference best practices: tailoring services to your library. Chicago: ALA, 2009, p. 32.

10. Kovacs, Diane K. The virtual reference handbook: interview and information delivery techniques for the chat and e-mail environments. London: Facet, 2007.

José-Antonio Merlo-Vega. Universidad de Salamanca. merlo@usal.es

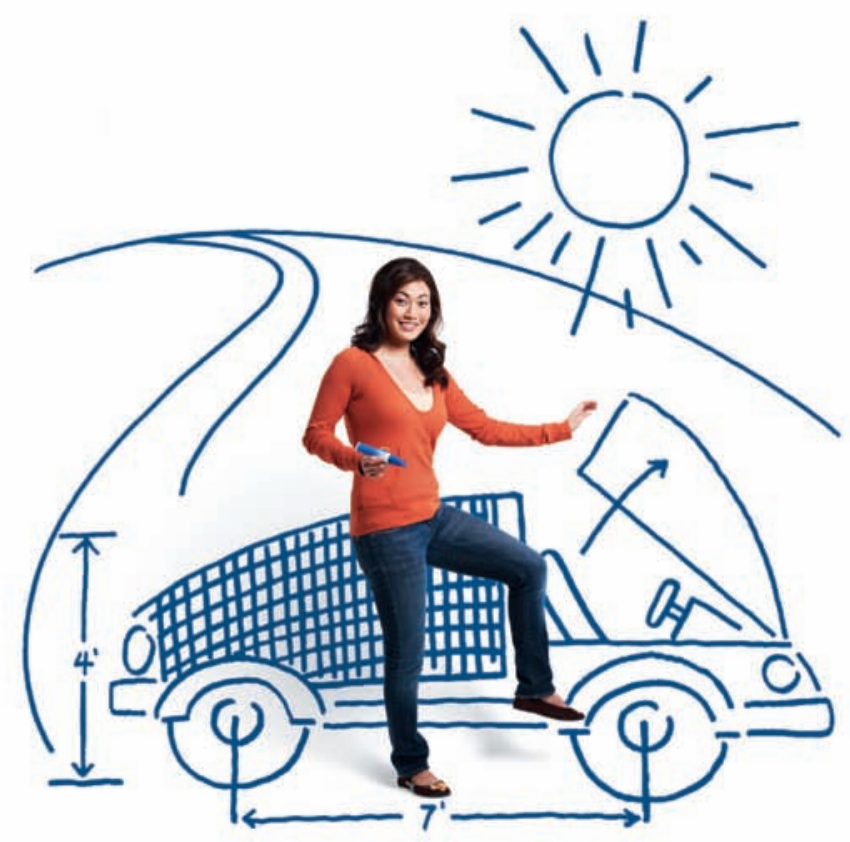

\section{Para conseguirlo, te necesitan.}

Como especialista en información, tu labor es mucho más que el punto de unión entre los usuarios y las publicaciones. Ayudas a encontrar la inspiración que necesita la gente para avanzar en sus investigaciones, para inventar lo que revolucionará el mundo o quizá para solucionar un problema global. EBSCO, líder mundial en servicios de información, puede ayudarte a hacerlo poniendo el contenido de 79.000 editores a tu disposición. Además, contarás con el apoyo de más de 130 bibliotecarios especializados. Con nuestros sistemas de gestión de la información podrás aprovechar mejor tu tiempo y atender mejor a tus usuarios. Después de todo, ¿quién sabe lo que el próximo genio te pedirá?

www.ebsco.es 


\section{ProQuest}

\section{Literature Online}

El sitio de referencia para la literatura y crítica literaria en inglés http://lion.chadwyck.co.uk
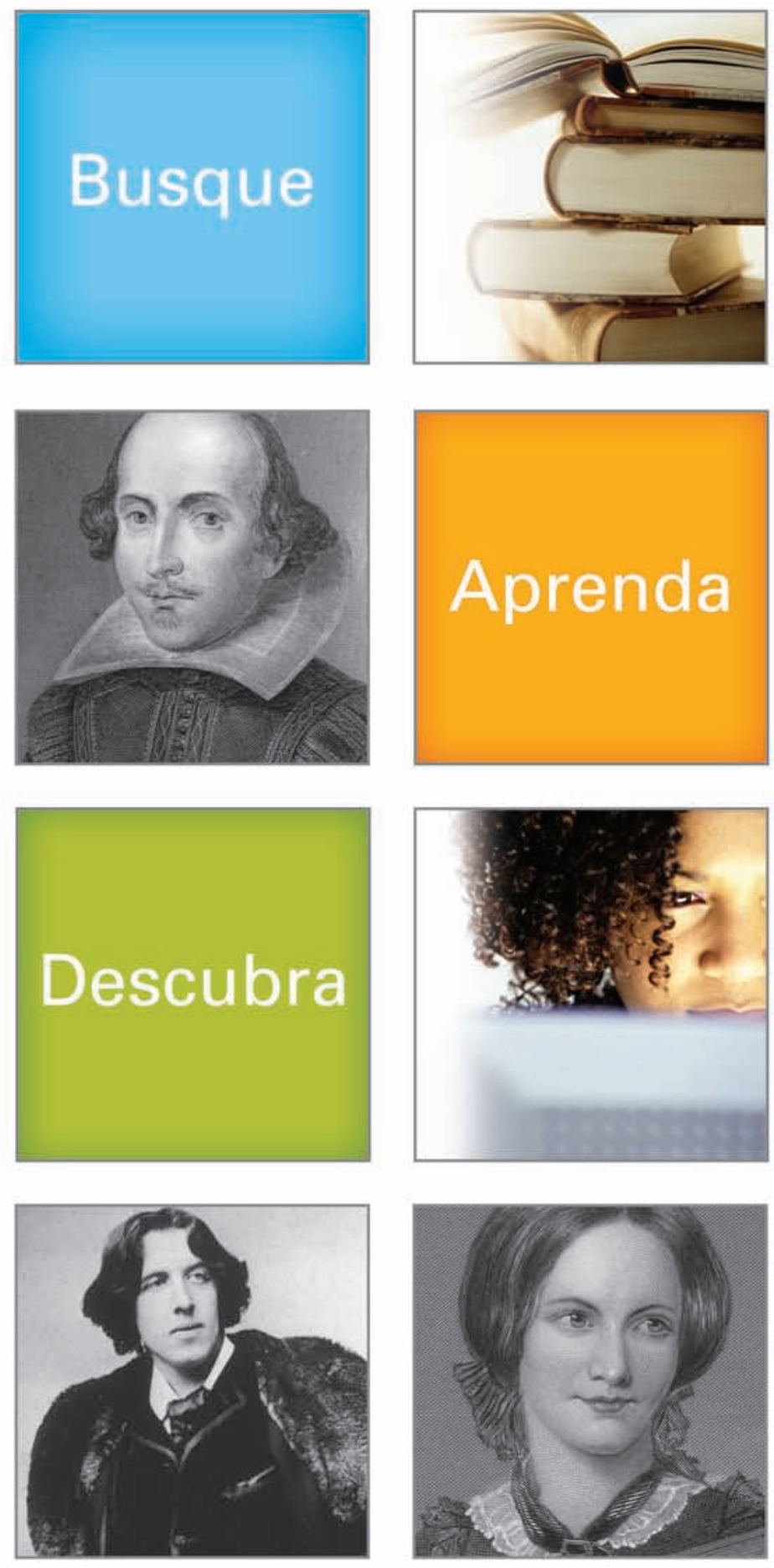

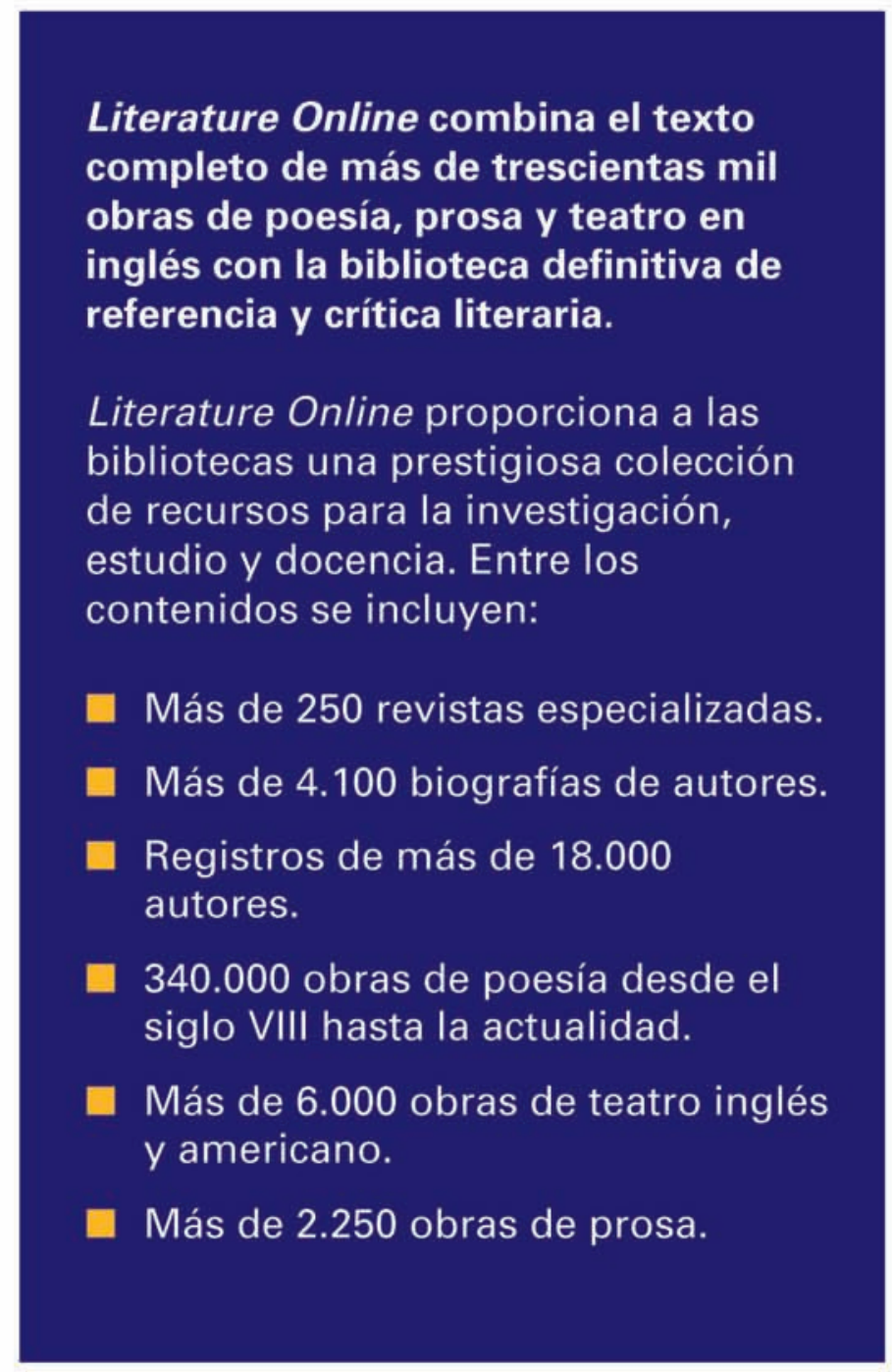

Para más información póngase en contacto con editor@spain.proquest.co.uk citando la referencia AD 83709. 


\section{Evolucionamos hacia nuevos desafíos}

Hemos recorrido un largo camino en la gestión de servicios bibliográficos, archivísticos y documentales, sin dejar de aprender y crecer.

La evolución tecnológica requiere nuevos servicios personalizados, capaces de integrar su información convencional en la nueva era digital.

Soluciones variadas y de calidad que aseguren la accesibilidad y el interés por sus colecciones.

Con la misma ilusión y profesionalidad ponemos a su alcance nuestros servicios de recursos digitales. Estará en buenas manos.

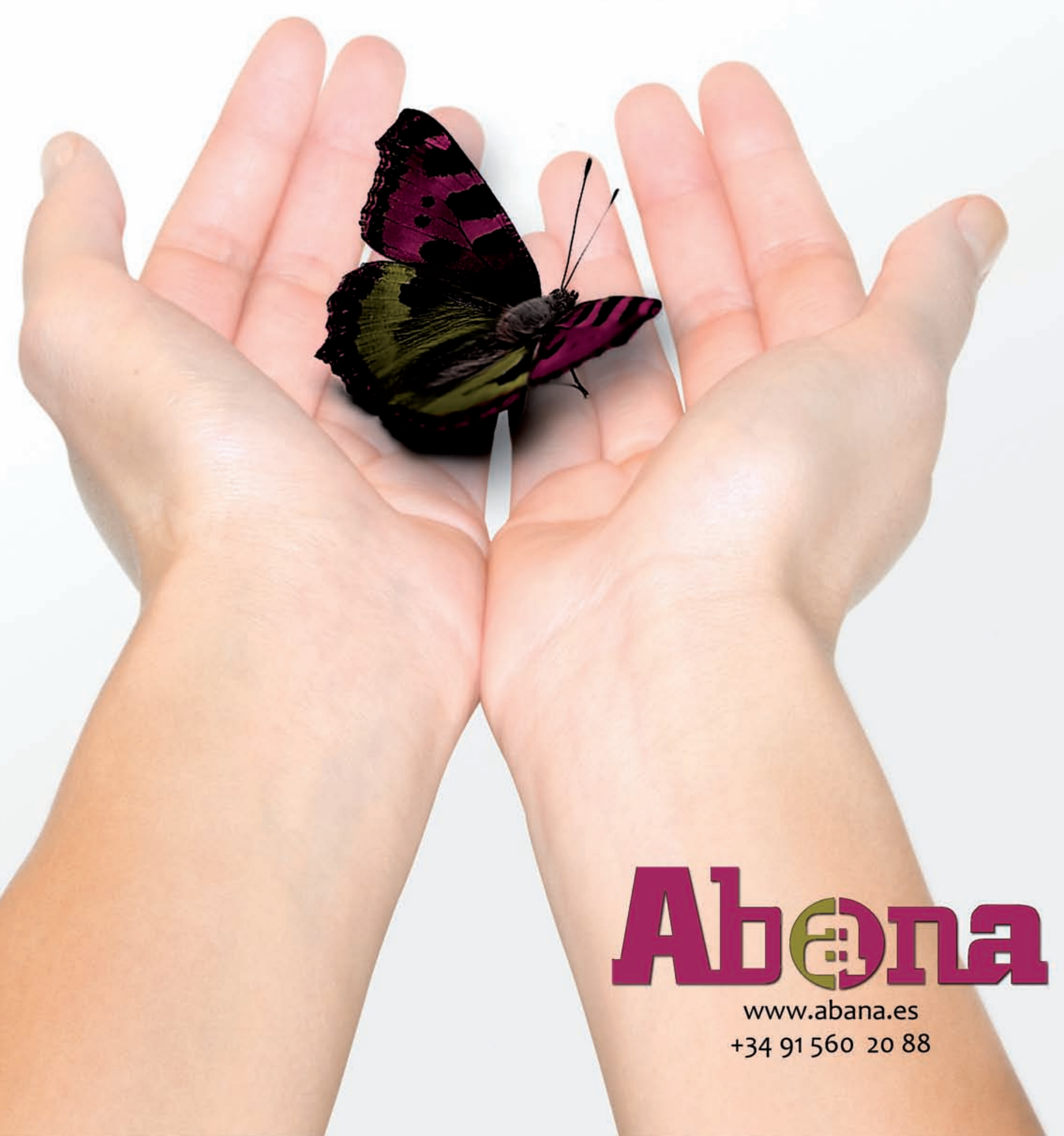

